\title{
A Morphometric And Ultrastructural Evaluation of Capillaries In Rabbit Vastus Lateralis Muscle Before and After Compressed Air Massage
}

*M.A. Gregory, and ^ M.Mars

*Electron Microscope Unit, University of Durban-Westville, Durban 4000, South Africa

^Physiology Dept., Nelson R. Mandela Medical School, University of Natal, Durban, South Africa

Massage, per-se, is thought to encourage the healing of soft tissue injuries by improving blood flow to the damaged region. Recent light microscopical, morphometric studies by this group have shown that capillary diameters are increased after two different massage techniques[1]. Although our results suggest that massage improves blood flow, the resolution of the light microscope does not enable endothelial cell morphology or lumenal area to be measured. Should endothelial cells swell after massage, it is possible that the apparent increase in capillary diameter may have been a consequence of endothelial cell oedema. Under such circumstances, capillary lumens, rather than increasing in diameter may have decreased, thereby potentially reducing blood flow. In order to determine the precise state of capillaries after massage, this study examines the fine-structure and morphometrics of capillaries before and up to 6 days after compressed air (JET®) massage.

Four New Zealand, white rabbits were studied (R1 - R4). Before massage, the animals were anaesthetised by an intramuscular injection of equal parts Ketamine and Xylazine $(10 \mathrm{mg} / \mathrm{kg})$ and the fur was removed from the skin over the left thigh. Treatment consisted of a single, 10 minute episode of JET therapy using the "sport", single hole $(4 \mathrm{~mm})$ head and unheated air at 1 bar. Wedge biopsies were taken from the left vastus lateralis within 10 minutes (R1 - R2) and 24 hours (R3R4) after treatment. To serve as controls, similar biopsies were taken from the right vastus lateralis of each animal. Tissue samples approximately $1 \mathrm{~mm} \mathrm{X} 2 \mathrm{~mm}$ were immediately placed in Karnovsky's fixative in $0.2 \mathrm{M}$ cacodylate buffer. Following dehydration and perfusion with Spurr's epoxy resin, the tissue was longitudinally orientated and "flat" embedded in silicone moulds. Thick, $(1 \mu \mathrm{m})$ transverse sections of muscle were cut and stained with $1 \%$ aqueous alkaline toluidine blue and areas containing cross-sectioned capillaries were selected for ultramicrotomy. Ultrathin sections, 60nm in thickness were cut and examined using a Jeol 1010 TEM. Images were obtained with a Kodak Megaplus camera and images evaluated using SIS software. At least 25 capillaries were collected from each specimen. Endothelial cell thickness was determined by calculating the mean of a minimum 20 observations from areas away from nuclei. The external and lumenal diameters were measured using the least diameter method[2] and cross-sectional areas calculated from these data. Statistical analyses were undertaken using the Krukal-Wallace ANOVA.

Other than an apparent thinning of capillary walls after massage, no pathomorphology was noted in any endothelial cells (Fig. 1). The morphometric results are summarised in the table and the overall change in capillary dimensions after 10 minutes and 24 hours of JET therapy is portrayed in the bar graph. Note that in all cases, both external and lumenal capillary diameters were increased for the duration of the 24 hour test period. The relationship between mean endothelial cell thickness and external diameter of control and JET treated tissue are described as linear regressions in Fig. 2. Note that for any given capillary diameter in control tissue, the endothelial cell thickness of capillaries after JET therapy is thinner. As there was no obvious ultrastructural pathology, such thinning is probably a consequence of capillary dilation. 
These results give credence to the view that massage effects repair of injured soft tissues by improving blood flow to the damaged region. To the best of our knowledge, this is the first report that shows that any type of massage provides such benefit for extended periods of time.

\section{References}

1. M.A.Gregory and Mars, Microsc. \& Microanalysis 9, (suppl.2 - submitted 2003)

2. V.Dubowitz, Muscle Biopsy: A practical approach. Bailliere Tindall, London, 1985

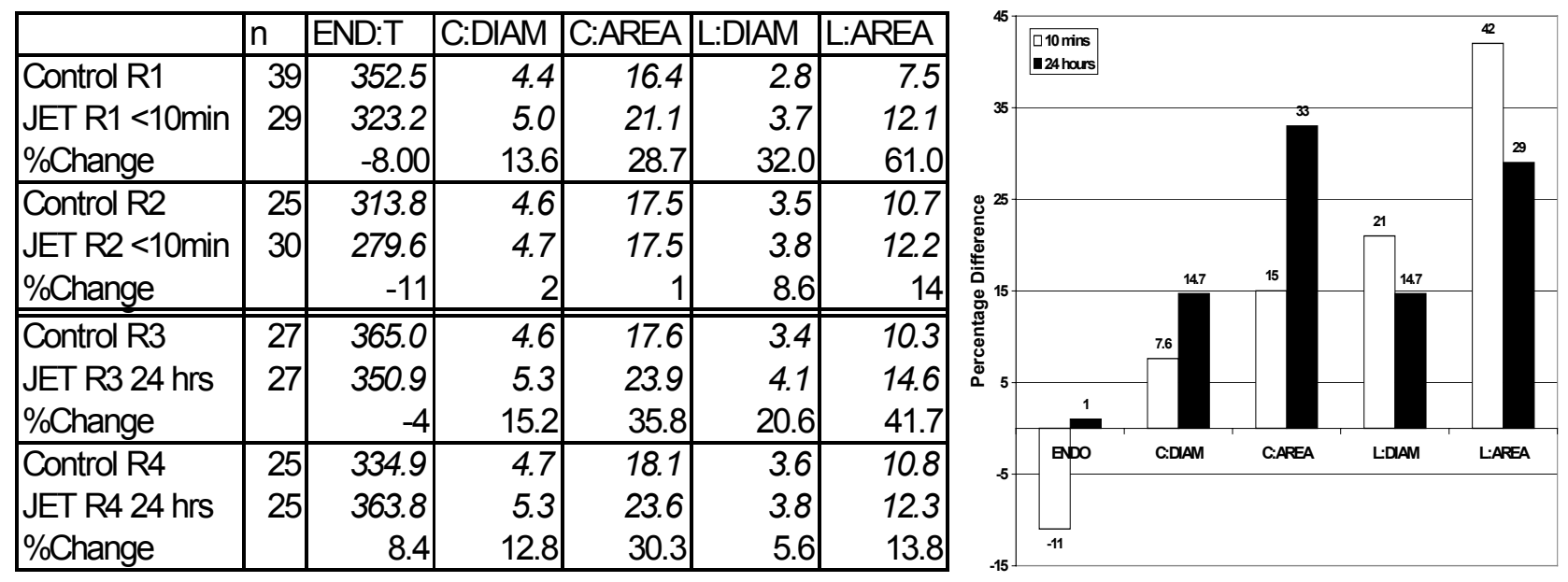
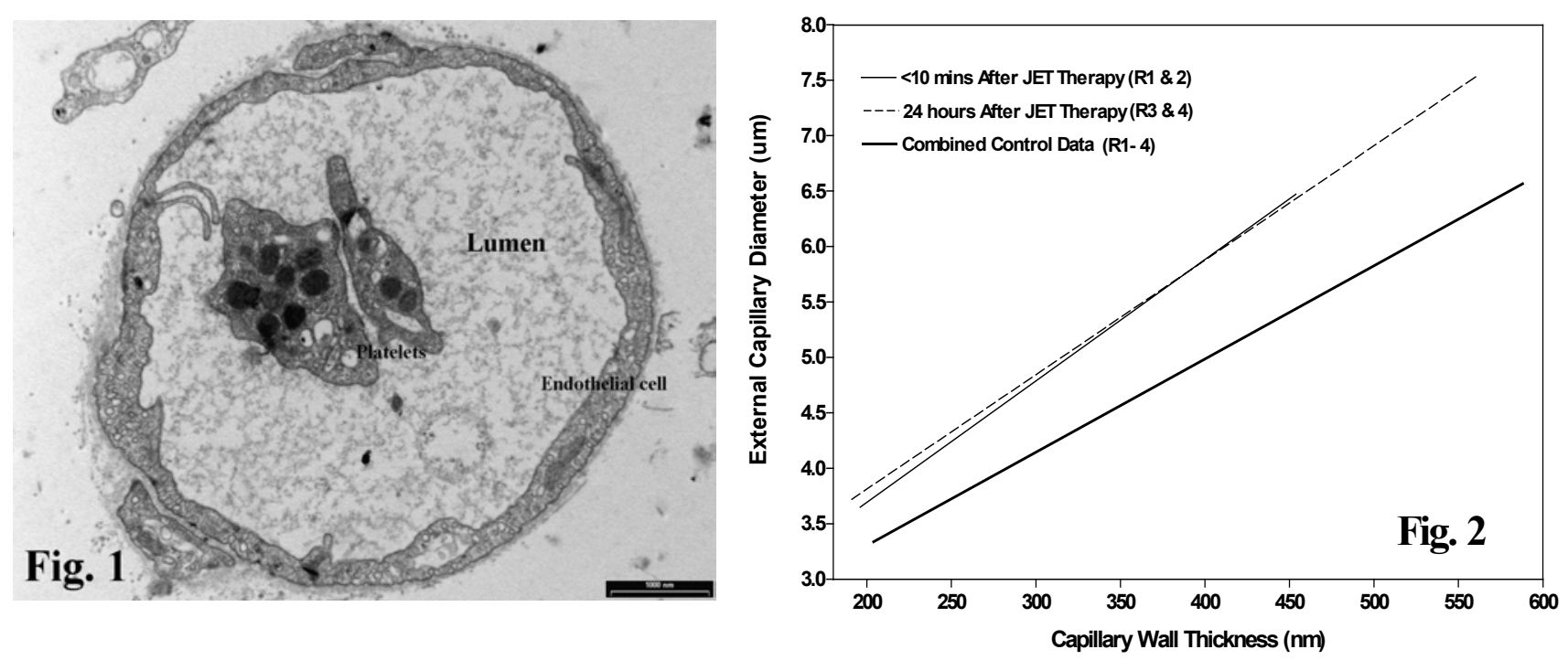

Table: Summary of morphometric data. $\mathrm{n}=$ number of measurements; END:T endothelial cell thickness $(\mathrm{nm}) \mathrm{C} \& \mathrm{~L}$ :DIAM = external capillary and lumenal diameters $(\mu \mathrm{m}) ; \mathrm{C} \& \mathrm{~L}: A R E A=$ external capillary and lumenal area $\left(\mu \mathrm{m}^{2}\right) ; \%$ change $=$ percentage change control v's JET. Bar Graph $=$ Mean percentage change in capillary external and lumenal diameters and areas. Fig. 1: Electron micrograph of cross-sectioned capillary from rabbit R1 (10 minutes post-JET). Fig. 2. Regressions showing the relationship between capillary wall thickness and capillary diameter. Note that for a given capillary diameter, wall thickness is thinner after JET therapy suggesting capillary dilation. 